\title{
Reduced High-order Aberrations using Wavefront-guided Partial Photorefractive Keratectomy and Accelerated Epithelium-off Corneal Cross-linking for Keratoconus
}

\author{
${ }^{1}$ Victor Penner, ${ }^{2}$ Kylee Lewis, ${ }^{3}$ Guillermo Rocha
}

\begin{abstract}
Purpose: To demonstrate clinical outcomes of epithelium-off corneal cross-linking $(\mathrm{CXL})$ in combination with wavefrontguided photorefractive keratectomy (PRK) for the treatment of keratoconuns.

Materials and methods: Total, 28 keratoconic eyes of 21 patients were included. The Sirius Wavefront Analyzer (SCHWIND eye-tech-solutions $\mathrm{GmbH}$ and Co. KG) was used to evaluate the high-order aberrations (HOAs). The coupled Amaris $750 \mathrm{~S}$ excimer laser then performed a PRK of no more than $40 \mu \mathrm{m}$ using the measured HOA. The CXL was then carried out respecting the standard of $400 \mu \mathrm{m}$ of corneal thickness.
\end{abstract}

Results: The average age was $32(15-48)$ years. Uncorrected distance visual acuity (UDVA) improved from 0.58 to 0.31 $\operatorname{logMAR}(p<0.001)$, with best corrected distance visual acuity (CDVA) improving from 0.03 to 0.02 logMAR $(p=0.7)$.

On manifest refraction, the average spherical component decreased from -1.87 to $-1.40 \mathrm{D}(0.47 \pm 2.12, p=0.4)$. Cylinder decreased from 2.18 to $0.87 \mathrm{D}(1.31 \pm 1.14 \mathrm{D}, \mathrm{p}<0.001)$.

All HOAs decreased: total $\mathrm{HO} 0.94$ to $0.58(0.36 \pm 0.42$, $p=0.05)$; spherical aberration: 0.142 to $-0.018(0.160 \pm 0.160$, $p<0.001)$; secondary astigmatism: 0.21 to 0.11 ( $0.10 \pm 0.11$, $\mathrm{p}=0.02)$; coma: 0.80 to $0.42(0.38 \pm 0.47, \mathrm{p}=0.03)$; trefoil: 0.33 to $0.29(0.04 \pm 0.17, p=0.7)$.

Conclusion: Utilizing HOA to guide the laser treatment in combination with $\mathrm{CXL}$ resulted in a significant decrease of $\mathrm{HOA}$ at 6 months postprocedure. This is a small case series, but demonstrates a promising trend of improved HOA. Considering that keratoconic eyes have very irregular surfaces with significant HOA, reducing these aberrations should improve best corrected quality of vision. Having longer follow-up and greater numbers may demonstrate a clearer improvement.

Keywords: Cornea, Ectasia, High-order aberrations, Keratoconus, Photorefractive keratectomy, Spherical aberrations, Trefoil.

How to cite this article: Penner V, Lewis K, Rocha G. Reduced High-order Aberrations using Wavefront-guided Partial Photorefractive Keratectomy and Accelerated Epithelium-off

\footnotetext{
${ }^{1}$ Resident, ${ }^{2}$ Medical Student, ${ }^{3}$ Associate Professor

${ }^{1,3}$ Department of Ophthalmology, Max Rady College of Medicine University of Manitoba, Winnipeg, Manitoba, Canada

${ }^{2}$ Department of Undergraduate Medicine, Max Rady College of Medicine, University of Manitoba, Winnipeg, Manitoba, Canada

Corresponding Author: Victor Penner, Resident, Department of Ophthalmology, Max Rady College of Medicine, University of Manitoba, Winnipeg, Manitoba, Canada, Phone: +12047888599 e-mail: victor.penner@gmail.com
}

Corneal Cross-linking for Keratoconus. Int J Kerat Ect Cor Dis 2017;6(2):73-77.

Source of support: Nil

Conflict of interest: None

\section{INTRODUCTION}

Keratoconus is a progressive, noninflammatory thinning of the cornea. The resulting irregular shape from this ectatic process is associated with significant irregular astigmatism and HOAs. The treatment goal of keratoconus consists of visual rehabilitation and addressing the progressive thinning. ${ }^{1,2}$

Optical correction with spectacles and contact lenses has been the mainstay of treatment to correct the significant refractive errors associated with keratoconus. In particular, rigid contact lenses neutralize the significant corneal irregularity present and, thereby, address the distorted vision associated with HOAs. Rigid lenses, however, can be challenging to fit, uncomfortable, and cause apical scarring. As the disease progresses, many patients become contact lens-intolerant. ${ }^{1}$

The first surgical attempt to alter the shape of the cornea was with placement of intrastromal ring segment (ICRS). ${ }^{3,4}$ While well tolerated, ICRS have a low precision in targeting specific refractive errors in individual cases. ${ }^{5}$ Implantable phakic intraocular lenses (IOLs) have also provided visual rehabilitation, but can only address regular astigmatism. With the advent of corneal $\mathrm{CXL}^{6}$ there have been further advances in the surgical management of refractive error through the concomitant use of excimer laser treatments to alter the shape of the cornea. ${ }^{7}$

The CXL has been developed over the past two decades as a technique to stabilize the cornea and prevent further progression. This revolutionary technique has demonstrated sufficient stability to allow the application of excimer laser to corneas previously thought to be too risky to treat due to the high risk of developing further ectasia. $^{8-10}$

Our previous work using epithelium-off CXL in combination with a bitoric (cross-cylinder) PRK ablation demonstrated a significant improvement in UDVA, CDVA, and flattening of the cornea (unpublished observations). 
However, it did not show a significant improvement in total HOAs. In this series, we aim to evaluate the effect of a wavefront-guided PRK when used in combination with epithelium-off CXL for the treatment of keratoconus.

\section{MATERIALS AND METHODS}

This study was a retrospective, nonrandomized, interventional case study involving patients who underwent wavefront-guided PRK combined with simultaneous same day epithelium-off CXL. All of the procedures took place between December 2013 and November 2015. Ethics approval was obtained through the University of Manitoba and written informed consent was obtained for the interventions in accordance with the Declaration of Helsinki.

For eyes to be included in the study, they had to have keratoconus with an Amsler-Krumeich classification of grades I or II. The diagnosis of keratoconus was established through clinical assessment and tomographic analysis of the cornea. Prior to surgery, all eyes were at risk of progression or had proven changes consistent with progression. Also, all patients had to have poor "satisfactory best-corrected" vision. ${ }^{1}$ This meant they could have good corrected vision. However, either the overall quality of their vision was poor or they could not tolerate the corrective lenses for extended periods of time. Additional inclusion criteria were: A minimum central corneal pachymetry of $440 \mu \mathrm{m}$; keratometry readings $\leq 55 \mathrm{D}$; and an estimated residual stromal thickness of at least $390 \mu \mathrm{m}$ after maximum excimer laser central stromal ablation of $40 \mu \mathrm{m}$ following epithelial removal. Exclusion criteria were previous corneal surgery; central corneal scarring; and pregnancy or nursing.

The primary outcome of the study was improved visual quality as defined by best corrected visual acuity and measured HOAs. Secondary outcomes included stabilization of keratoconus and halting progression.

\section{Measurements}

All patients were evaluated preoperatively with a full examination including UDVA, CDVA, manifest refraction, slit-lamp exam, tonometry, and fundoscopy. Visual acuity was measured using the ClearChart 2 Digital Acuity System (Reichert Technologies; Depew, NY) with logMAR optotype display. All examinations and manifest refraction were performed by one examiner (GR). The anterior segment was assessed with optical coherence tomography to evaluate epithelial thickness (RTVue; Fremont, CA). Further Placido-based topography and total ocular aberrometry (iTrace ray tracing aberrometer/ topography; Tracey Technologies, Houston, TX), in addition to Scheimpflug corneal tomography (Pentacam HF;
Oculus GmbH, Wetzlar, Germany) were obtained. All HOAs were assessed at a 5-mm pupil.

\section{Surgical Planning}

Three steps were used to individualize the treatment parameters for each eligible eye:

1. The Sirius Wavefront Analyzer (SCHWIND eye-techsolutions $\mathrm{GmbH}$ and Co. $\mathrm{KG}$ ) was used to evaluate the corneal HOA.

2. Estimation of residual stromal thickness: The goal was to have an estimated residual stromal thickness of at least $390 \mu \mathrm{m}$ (preferably $400 \mu \mathrm{m}$ ) after a maximum excimer laser central stromal ablation of $40 \mu \mathrm{m}$ following epithelial removal.

3. Planning was achieved using the management software, minimizing depth, volume, and HOA as required.

\section{Surgical Technique}

All surgeries were performed by the same surgeon (GR). Laser treatment parameters were entered into the Amaris $750 S$ (SCHWIND eye-tech-solutions $\mathrm{GmbH}$ and Co. $\mathrm{KG}$ ). Under topical anesthesia, a 9.0-mm epithelial area was debrided with a surgical brush (Hyperopic Amoils Epithelial Scrubber; Innovative Excimer Solutions Inc., Toronto, ON). A partial PRK ablation then followed. The intent was to minimize astigmatism and HOAs, reduce the spherical correction to around $-1.00 \mathrm{D}$, and respect a maximum ablation of $40 \mu \mathrm{m}$, leaving a residual stromal thickness of at least $390 \mu \mathrm{m}$ at the center. Following the ablation, irrigation was carried out with balanced salt solution (BSS), and application of mitomycin-C $0.02 \%$ for 12 seconds, with subsequent copious irrigation using $10 \mathrm{~mL}$ of BSS.

The CXL procedure was slightly modified from that initially described by Wollensak et al. ${ }^{6}$ In brief, pilocarpine $2 \%$ was instilled. Riboflavin $0.1 \%$ in dextran solution was applied every 2 minutes for 30 minutes. When necessary, hypotonic riboflavin solution was used to ensure a corneal thickness of at least $400 \mu \mathrm{m}$ before initiating UVA irradiation. The debrided area was then exposed to $370 \mathrm{~nm}$ wavelength UVA irradiation $/ 9 \mathrm{~mW} / \mathrm{cm}^{2}$ for 10 minutes, while instillation of riboflavin continued in an alternating fashion every minute (dextran/hypotonic). Following the procedure, topical antibiotic, steroid, and cycloplegic drops were instilled, and a bandage contact lens was placed.

Postoperatively, topical moxifloxacin hydrochloride 0.5\% (Vigamox, Alcon Laboratories, Ft. Worth, TX) was prescribed QID until epithelial closure; dexamethasone 0.1\% (Maxidex, Alcon Laboratories, Ft. Worth, TX) was prescribed QID and tapered weekly over a month, and frequent artificial tears were prescribed. The bandage 
contact lens was removed upon epithelial closure between 5 and 7 days postoperatively.

\section{Postoperative Assessment}

A complete examination was performed postoperatively including UDVA, CDVA, refraction, and slit-lamp assessment. Placido-based topography, total ocular aberrometry, and Scheimpflug corneal tomography were repeated at the 6 month follow-up.

\section{Primary Outcomes}

UDVA, CDVA, and HOAs.

\section{Statistical Analysis}

Analysis was carried out with the paired two-tailed Student's t-test. Significance level was established at $\mathrm{p}<0.05$. For quantitative data, the mean \pm standard deviation was reported and nominal data were expressed as a percentage.

Table 1: Patient demographics

\begin{tabular}{ll}
\hline Number of eyes (number of patients) & $28(21)$ \\
Right eyes (left eyes) & $13(15)$ \\
Male (female) & $19(9)$ \\
Average age & 32 \\
Median age & 30 \\
Age range & $15-48$ \\
\hline
\end{tabular}

Table 3: CDVA: Percent eyes at each acuity level comparing pre- and postoperatively

\begin{tabular}{lll}
\hline & Preoperative & Postoperative \\
\hline $20 / 32$ & 5 & 0 \\
$20 / 25$ & 31 & 32 \\
$20 / 20$ & 50 & 54 \\
$20 / 16$ & 14 & 14 \\
\hline
\end{tabular}

Table 5: Pre- and postoperative manifest refraction (Diopters)

\begin{tabular}{lrrllll}
\hline & \multicolumn{5}{c}{ 6-month data $(n=28)$} \\
\cline { 2 - 7 } & Pre & $6 m$ & Diff & $S D$ & $C l$ & $p$-value \\
\hline Sphere & -1.87 & -1.40 & 0.47 & \pm 2.12 & \pm 0.88 & 0.4 \\
Cyl & 2.18 & 0.87 & 1.31 & \pm 1.14 & \pm 0.47 & $<0.001$ \\
\hline
\end{tabular}

SD: Standard deviation; $\mathrm{Cl}$ : Confidence interval

\section{RESULTS}

In total, 28 eyes of 21 patients were included in the analysis (Table 1). All patients had complete preoperative and 6-month follow-up as described in the methods.

Both UDVA and CDVA improved at 6 months; however, only UDVA improved significantly. As can be seen in Table 2, the preoperative CDVA was $0.03 \log$ MAR prior to surgery with minimal change (0.02 logMAR) postoperatively. No eyes lost any lines of vision with $58 \%$ of eyes achieving a UDVA $20 / 32$ or better (Tables 3 and 4$)$.

The manifest refraction became less myopic by an average of $0.47 \mathrm{D}$ with a significant decrease in cylindrical correction (1.31 D improvement; Table 5). The simulated steep and flat keratometry readings flattened an average of 0.9 and $0.6 \mathrm{D}$ respectively (Table 6).

Postoperative total HOAs, in addition to coma, spherical aberration, and secondary astigmatism all had a significant reduction, with trefoil demonstrating improvement not reaching significance (Table 7).

Table 2: Average change in UDVA and CDVA at 6 months postoperatively (logMAR visual acuity)

\begin{tabular}{llllllc}
\hline & \multicolumn{6}{c}{ 6-month data $(n=28)$} \\
\cline { 2 - 7 } & Pre & $6 m$ & Diff & $S D$ & Cl & $p$-value \\
\hline UDVA & 0.58 & 0.31 & 0.27 & \pm 0.33 & \pm 0.13 & $<0.01$ \\
CDVA & 0.03 & 0.02 & 0.01 & \pm 0.09 & \pm 0.04 & 0.68 \\
\hline
\end{tabular}

SD: Standard deviation; $\mathrm{Cl}$ : Confidence interval

Table 4: UDVA: Percent eyes at each acuity level comparing pre- and postoperatively

\begin{tabular}{lll}
\hline & Preoperative & Postoperative \\
\hline $20 / 40$ or worse & 92 & 42 \\
$20 / 32$ or better & 8 & 58 \\
\hline
\end{tabular}

Table 6: Pre- and postoperative flat and steep simulated keratometry from Scheimpflug imaging

\begin{tabular}{lllllll}
\hline & \multicolumn{6}{c}{ 6-month data $(n=28)$} \\
\cline { 2 - 7 } & Pre & $6 m$ & Diff & $S D$ & Cl & p-value \\
\hline $\mathrm{K}_{\min }$ (D) & 43.4 & 42.8 & 0.6 & \pm 2.7 & \pm 1.2 & 0.4 \\
$\mathrm{~K}_{\max }$ (D) & 46.1 & 45.2 & 0.9 & \pm 1.7 & \pm 0.8 & 0.3 \\
\hline
\end{tabular}

SD: Standard deviation; $\mathrm{Cl}$ : Confidence interval

Table 7: Pre- and postoperative HOAs. Note only 18 eyes were included as some eyes did not have pupil size large enough

\begin{tabular}{lllllll}
\hline & \multicolumn{5}{c}{ 6-month data $(n=18)$} \\
\cline { 2 - 7 } & Pre & $6 m$ & Diff & $S D$ & $\mathrm{Cl}$ & $p$-value \\
\hline Total RMS, $\mu \mathrm{m}$ & 0.94 & 0.58 & 0.36 & \pm 0.42 & \pm 0.23 & 0.05 \\
Coma, $\mu \mathrm{m}$ & 0.80 & 0.42 & 0.38 & \pm 0.47 & \pm 0.26 & 0.03 \\
Primary spherical aberration, $\mu \mathrm{m}$ & 0.142 & -0.018 & 0.160 & \pm 0.103 & \pm 0.056 & $<0.001$ \\
2nd astig, $\mu \mathrm{m}$ & 0.21 & 0.11 & 0.10 & \pm 0.11 & \pm 0.06 & 0.02 \\
Trefoil, $\mu \mathrm{m}$ & 0.33 & 0.29 & 0.04 & \pm 0.17 & \pm 0.09 & 0.7 \\
\hline
\end{tabular}

SD: Standard deviation; Cl: Confidence interval 


\section{DISCUSSION}

In our approach to keratoconus, we employ a very logical and consistent approach. This is to done to identify which patients benefit from the different treatment options available. Our treatment decisions are based on three clinical questions: Is there progression? Does shape of the cornea need to be altered? Does the quality of vision need to be improved? If the patient can achieve satisfactory corrected vision with spectacles or contact lenses and is not at risk of progression or does not demonstrate any progression, no further surgical intervention needs to be considered. However, if progression is a concern, the cornea needs to be stabilized and CXL is one of the treatment modalities offered. If the corneal shape is abnormal, treatment options of ICRS, bitoric PRK, wave-guided PRK can be considered.

The CXL has been demonstrated to stabilize the cornea and halt progression as is well summarized by Ziaei et al. ${ }^{2}$ The CXL has also been combined with other surgical approaches to help improve the corneal shape and vision. One option is to combine CXL with implantation of ICRS. ${ }^{3}$ This technique not only improves corneal contour, but also improves the patient's quality of vision. A second option is to treat the corneal surface with a topography-guided custom ablation treatment (T-CAT) followed by CXL as described in the Athens Protocol. ${ }^{7}$ This technique improves not only corneal contour, but also visual acuity. It has also been demonstrated to be safe with stability and no progression by Kanellopoulos et $\mathrm{al}^{11}$ in a 3-year follow-up study. ${ }^{12}$ While reducing the corneal irregularity, T-CAT, however, does not target the visual distortion caused by HOAs directly.

When trying to improve visual performance and optical system of the eye, the next logical area to focus would be the HOAs. A reduction in HOAs has been demonstrated to improve contrast sensitivity testing, ${ }^{13}$ and, in particular, it has been demonstrated that keratoconic patients would benefit from a reduction in HOAs. ${ }^{14}$ When assessing HOAs and CXL alone, there are modest improvements, but no consistent significant changes. ${ }^{15}$ There are now multiple different attempts to refine treatment protocols with different ablation patterns and objectives. To date, most protocols are targeting the topography of the cornea in order to improve the contour and, hence, visual quality. ${ }^{16}$ While there is often an improvement in HOAs with topography-guided ablation profiles, our previous unpublished work finds these changes do not reach statistical significance. However, there are some other series that find a scattering of significant improvements in trefoil or coma. ${ }^{12,17-19}$ Of note, coma seems to contribute to the largest component of the total HOAs in keratoconus. ${ }^{15,20-22}$ Knowing this, coma was given the most weight in the planning of the ablation profile.

Overall, our results demonstrated stability or improvement in all the parameters measured. The UDVA improved significantly from 0.58 to $0.31 \log$ MAR. The CDVA remained stable with no patients losing any lines of CDVA or UDVA. Most patients had a reasonable CDVA prior to the procedure and there was little room to improve on a standard visual acuity chart; this is likely the explanation as to why the CDVA remained unchanged from the preoperative measurements. On manifest refraction, the spherical component remained largely stable, but there was a dramatic drop in average cylinder from 2.18 to $0.87 \mathrm{D}$. In this current series, our results demonstrated significant reduction in total, coma, spherical aberration and secondary astigmatism, with an improvement in trefoil.

These results are similar to the results achieved by Shaheen et $\mathrm{al}^{23}$ in their case series of wave-guided PRK and CXL, which demonstrated a significant reduction in total, coma, and trefoil, and an improvement in spherical aberration. Shaheen et $\mathrm{al}^{23}$ however, differed in their approach in that the wave-guided PRK was applied to keratoconic eyes that had previously undergone CXL rather than performing the procedures simultaneously. It is re-assuring to see similarly significant improvements in HOAs in both delayed and simultaneous approaches. This, however, is a potential area of further comparison and research. Potentially more accurate results could be obtained in the simultaneous approach as the ablation is being carried out on a "virgin" eye, whereas performing the ablation on a previously cross-linked could be less predictable.

The limitations of this article are the lack of control group, with limited number of participants and only 6 months of follow-up. To further improve the understanding and significance of targeting HOAs in the ablation profile of a combined PRK and CXL procedure, a controlled study with longer follow-up would provide greater weight. Also, the use of high-contrast acuity charts is well known to be a limitation in comparing real-world visual performance. An alternative objective measure, such as contrast sensitivity or similar test could provide additional information as to the benefit of targeting HOAs.

\section{CONCLUSION}

Patients are not only seeking management of their ocular disease, but also improvement in the quality of their vision. This case series demonstrates that combining CXL with a wave-guided PRK does exactly that. By targeting HOAs with the PRK component of the treatment, patients' vision can be maximized by reducing the distorting effects of the aberrations. 
This is an exciting new step in keratoconus management. This is obviously a small case series and further evaluation with longer follow-up is needed to validate this procedure. The ramification for patients, however, is very important. Gaining improvement in quality of vision could allow patients to become more functional and less reliant on contact lenses.

\section{CLINICAL SIGNIFICANCE}

This would be the first published series of simultaneous wavefront-guided PRK and CXL. This has the potential not only to stabilize keratoconic corneas, but also to drastically improve the quality of vision. This is something that has been absent in previous treatment modalities.

\section{REFERENCES}

1. Gomes JA, Tan D, Rapuano CJ, Belin MW, Ambrosio R Jr., Guell JL, Malecaze F, Nishida K, Sangwan VS; Group of Panelists for the Global Delphi Panel of Keratoconus and Ectatic Diseases. Global consensus on keratoconus and ectatic diseases. Cornea 2015 Apr;34(4):359-369.

2. ZiaeiM,Barsam A,ShamieN, VromanD,Kim T, DonnenfeldED, Holland EJ, Kanellopoulos J, Mah FS, Randleman JB, et al. Reshaping procedures for the surgical management of corneal ectasia. J Cataract Refract Surg 2015 Apr;41(4):842-872.

3. Colin J, Cochener B, Savary G, Malet F. Correcting keratoconus with intracorneal rings. J Cataract Refract Surg 2000 Aug;26(8):1117-1122.

4. Ertan A, Colin J. Intracorneal rings for keratoconus and keratectasia. J Cataract Refract Surg 2007 Jul;33(7):1303-1314.

5. Alfonso JF, Lisa C, Fernandez-Vega L, Madrid-Costa D, Montes-Mico R. Intrastromal corneal ring segment implantation in 219 keratoconic eyes at different stages. Graefes Arch Clin Exp Ophthalmol 2011 Nov;249(11):1705-1712.

6. Wollensak G, Spoerl E, Seiler T. Riboflavin/ultraviolet-ainduced collagen crosslinking for the treatment of keratoconus. Am J Ophthalmol 2003 May;135(5):620-627.

7. Kanellopoulos AJ, Binder PS. Collagen cross-linking (CCL) with sequential topography-guided PRK: a temporizing alternative for keratoconus to penetrating keratoplasty. Cornea 2007 Aug;26(7):891-895.

8. Stojanovic A, Zhang J, Chen X, Nitter TA, Chen S, Wang Q. Topography-guided transepithelial surface ablation followed by corneal collagen cross-linking performed in a single combined procedure for the treatment of keratoconus and pellucid marginal degeneration. J Refract Surg 2010 Feb;26(2):145-152.

9. Kymionis GD, Kontadakis GA, Kounis GA, Portaliou DM, Karavitaki AE, Magarakis M, Yoo S, Pallikaris IG. Simultaneous topography-guided PRK followed by corneal collagen cross-linking for keratoconus. J Refract Surg 2009 Sep;25(9):S807-S811.
10. Mukherjee AN, Selimis V, Aslanides I. Transepithelial photorefractive keratectomy with crosslinking for keratoconus. Open Ophthalmol J 2013 Oct;7:63-68.

11. Kanellopoulos AJ, Asimellis G. Keratoconus management: long-term stability of topography-guided normalization combined with high-fluence CXL stabilization (the Athens Protocol). J Refract Surg 2014 Feb;30(2):88-93.

12. Alessio G, L'Abbate M, Sborgia C, La Tegola MG. Photorefractive keratectomy followed by cross-linking versus crosslinking alone for management of progressive keratoconus: two-year follow-up. Am J Ophthalmol 2013 Jan;155(1):54. e1-65.e1.

13. Piers PA, Manzanera S, Prieto PM, Gorceix N, Artal P. Use of adaptive optics to determine the optimal ocular spherical aberration. J Cataract Refract Surg 2007 Oct;33(10):1721-1726.

14. Williams D, Yoon GY, Porter J, Guirao A, Hofer H, Cox I. Visual benefit of correcting higher order aberrations of the eye. J Refract Surg 2000 Sep-Oct;16(5):S554-S559.

15. Wisse RP, Gadiot S, Soeters N, Godefrooij DA, Imhof SM, van der Lelij A. Higher-order aberrations 1 year after corneal collagen crosslinking for keratoconus and their independent effect on visual acuity. J Cataract Refract Surg 2016 Jul;42(7):1046-1052.

16. Pahuja NK, Shetty R, Sinha Roy A, Thakkar MM, Jayadev C, Nuijts RM, Nagaraja H. Laser vision correction with Q factor modification for keratoconus management. Curr Eye Res 2017 Apr;42(4):542-548.

17. Shaheen MS, El-Kateb M, Hafez TA, Pinero DP, Khalifa MA. Wavefront-guided laser treatment using a high-resolution aberrometer to measure irregular corneas: a pilot study. J Refract Surg 2015 Jun;31(6):411-418.

18. Shetty R, Nuijts RM, Nicholson M, Sargod K, Jayadev C, Veluri H, Sinha Roy A. Cone location-dependent outcomes after combined topography-guided photorefractive keratectomy and collagen cross-linking. Am J Ophthalmol 2015 Mar;159(3):419.e2-425.e2.

19. Pinero DP, Alio JL, Aleson A, Escaf M, Miranda M. Pentacam posterior and anterior corneal aberrations in normal and keratoconic eyes. Clin Exp Optom 2009 May;92(3):297-303.

20. Koller T, Iseli HP, Donitzky C, Ing D, Papadopoulos N, Seiler T. Topography-guided surface ablation for forme fruste keratoconus. Ophthalmology 2006 Dec;113(12):2198-2202.

21. Kymionis GD, Portaliou DM, Kounis GA, Limnopoulou AN, Kontadakis GA, Grentzelos MA. Simultaneous topographyguided photorefractive keratectomy followed by corneal collagen cross-linking for keratoconus. Am J Ophthalmol 2011 Nov;152(5):748-755.

22. Alio JL, Shabayek MH. Corneal higher order aberrations: a method to grade keratoconus. J Refract Surg 2006 Jun;22(6): 539-545.

23. Shaheen MS, Shalaby Bardan A, Pinero DP, Ezzeldin H, El-Kateb M, Helaly H, Khalifa MA. Wave front-guided photorefractive keratectomy using a high-resolution aberrometer after corneal collagen cross-linking in keratoconus. Cornea 2016 Jul;35(7):946-953. 